Estudios Geológicos, 68(2)

julio-diciembre 2012, 277-285

ISSN: 0367-0449

doi:10.3989/egeol.40472.147

\title{
Campamento Vespucio, una nueva localidad con mamíferos pleistocenos en la provincia de Salta, Argentina
}

\author{
Campamento Vespucio, a new locality with Pleistocene mammals \\ from Salta province, Argentina
}

\author{
G.G. Zacarías ${ }^{1}$, A.R. Miño-Boilini², A.E. Zurita², A. Francia²
}

\begin{abstract}
RESUMEN
En la presente contribución damos a conocer una nueva localidad con mamíferos fósiles pleistocenos, provenientes de una región poco explorada. Los materiales fueron hallados en la Quebrada del Aguay, en Campamento Vespucio, localidad de General Mosconi, provincia de Salta, Argentina, y tentativamente asignados a la Fm. Urundel (Pleistoceno tardío). Desde un punto de vista taxonómico, se presentan seis taxones que incluyen: Xenarthra Glyptodontidae: Glyptodon sp. y Panochthidae: Neosclerocalyptus sp.; Tardigrada Megatheriinae: gen. et. sp. indet; Proboscidea Gomphotheriidae: Stegomastodon sp; Notoungulata Toxodontidae: Toxodon sp.; Perissodactyla Equidae: Equus (Amerhippus) $\mathrm{sp}$. Este elenco faunístico se encuentra representado por taxones que presentan amplia distribución latitudinal en América del Sur. Algunos de ellos (e.g. Neosclerocalyptus) son indicadores de climas áridos/semiáridos y fríos de ambientes abiertos. Por último, este trabajo contribuye a mejorar la caracterización paleofaunística del Pleistoceno del norte de Argentina, muy poco conocida con respecto a aquella de la región Pampeana.
\end{abstract}

Palabras clave: Cuaternario, megafauna, Noroeste de Argentina.

\section{ABSTRACT}

In this contribution a new locality with Pleistocene fossil mammals is presented. The materials come from the Quebrada del Aguay, Campamento Vespucio, General Mosconi, Salta province, Argentina; they are tentatively assigned to the Urundel Fm. (late Pleistocene). The palaeofaunistic association includes six taxa: Xenarthra Glyptodontidae: Glyptodon sp. and Panochthidae: Neosclerocalyptus sp.; Tardigrada Megatheriinae: gen. et. sp. indet.; Proboscidea Gomphotheriidae: Stegomastodon sp; Notoungulata Toxodontidae: Toxodon sp. Perissodactyla Equidae: Equus (Amerhippus) sp. This set of Pleistocene mammals presents a wide latitudinal distribution in South America. Some of them (e.g. Neosclerocalyptus) are congruent with arid/semiarid, cold conditions and open environments. In this scenario, this contribution improves the knowledge that we have in northern part of Argentina, very scarce compared with respect to that of the Pampean region.

Key words: Quaternary, megafauna, northwestern Argentina.

\section{Introducción}

Los estudios referidos a mamíferos fósiles pleistocenos en el norte de la Argentina (Zurita et al., 2009) son muy escasos, comparados con aquellos efectuados en la región Pampeana (Tonni et al., 1999; Cione \& Tonni, 2005). El territorio de la provincia de Salta no escapa a dicha generalización. De este modo, se pueden citar solamente los aportes de Esteban et al. (1992), quienes dieron a cono-

\footnotetext{
1 Secretaría General de Ciencia y Técnica, Universidad Nacional del Nordeste, Corrientes, Argentina. Email: gzpaleozacarias@gmail.com 2 Centro de Ecología Aplicada del Litoral (CECOAL-CONICET) y Universidad Nacional del Nordeste. Ruta 5, km 2,5 (3400), Corrientes, Argentina. Email: angelmioboilini@yahoo.com.ar, aezurita74@yahoo.com.ar, analiafrancia@yahoo.com.ar
} 
cer los primeros registros de Scelidotherium Owen, 1839 para esta provincia, que incluyeron también una descripción litológica del sitio en donde fue exhumado el ejemplar. El material proviene de la localidad de Rosario de la Frontera ubicada a $118 \mathrm{~km}$ al sureste de la ciudad capital de Salta. Por otro lado, un estudio con mayor detalle estratigráfico fue llevado a cabo por Carlini et al. (1999) en el que dieron a conocer un conjunto de xenartros del Valle de Lerma y Sierra de Mojotoro. Estos materiales fueron exhumados de tres localidades, La Caldera, Campo Alegre y La Quesera. Finalmente, Zurita et al. (2002) comunicaron la presencia de Neosclerocalyptus Paula Couto, 1957 (Glyptodontidae), proveniente de Las Lajitas, incluyendo una breve descripción litológica del lugar de donde fue exhumado.

En este marco, el objetivo principal de esta contribución es dar a conocer un conjunto de mamíferos pleistocenos registrados en la localidad de Vespucio, General Mosconi, provincia de Salta, y de este modo ampliar el conocimiento de la paleofauna presente en el noroeste del actual territorio de la Argentina.

\section{Materiales y métodos}

\section{Área de estudio}

Los fósiles fueron colectados por campañas realizadas en colaboración entre el personal del Museo del Petróleo "Profesor Rodolfo Parodi Bustos" (MUPET- Vespucio) y la Fundación Miguel Lillo (provincia de Tucumán), en la Quebrada del Aguay, Vespucio, General Mosconi, provincia de Salta, Argentina (Fig. 1), durante los años 1980, 1983 y 1986. Actualmente estos materiales se encuentran depositados en la colección paleontológica de dicho Museo. Desde un punto de vista cronoestratigráfico se siguieron los esquemas propuestos por Cione y Tonni (1999; 2005).

Abreviaturas institucionales. PZ-MUPET: Paleozoología Museo del Petróleo, Salta, Argentina.

Abreviaturas anatómicas. M, m: molar superior e inferior, respectivamente.

\section{Contexto geológico}

La Quebrada del Aguay se encuentra dentro de las Sierras Subandinas. De acuerdo con Argollo \& Iriondo (2008) e Iriondo (2010) éstas forman una faja paralela de escasa altitud, ubicada al este de la Cordillera Oriental de Argentina. Dichas sierras tienen una extensión este-oeste de 60 a $80 \mathrm{~km}$ con una longitud aproximada de $180 \mathrm{~km}$, desde los actuales territorios de Bolivia y Perú hasta el norte de la provincia de Tucumán (Argentina); forma el frente montañoso andino contra los llanos

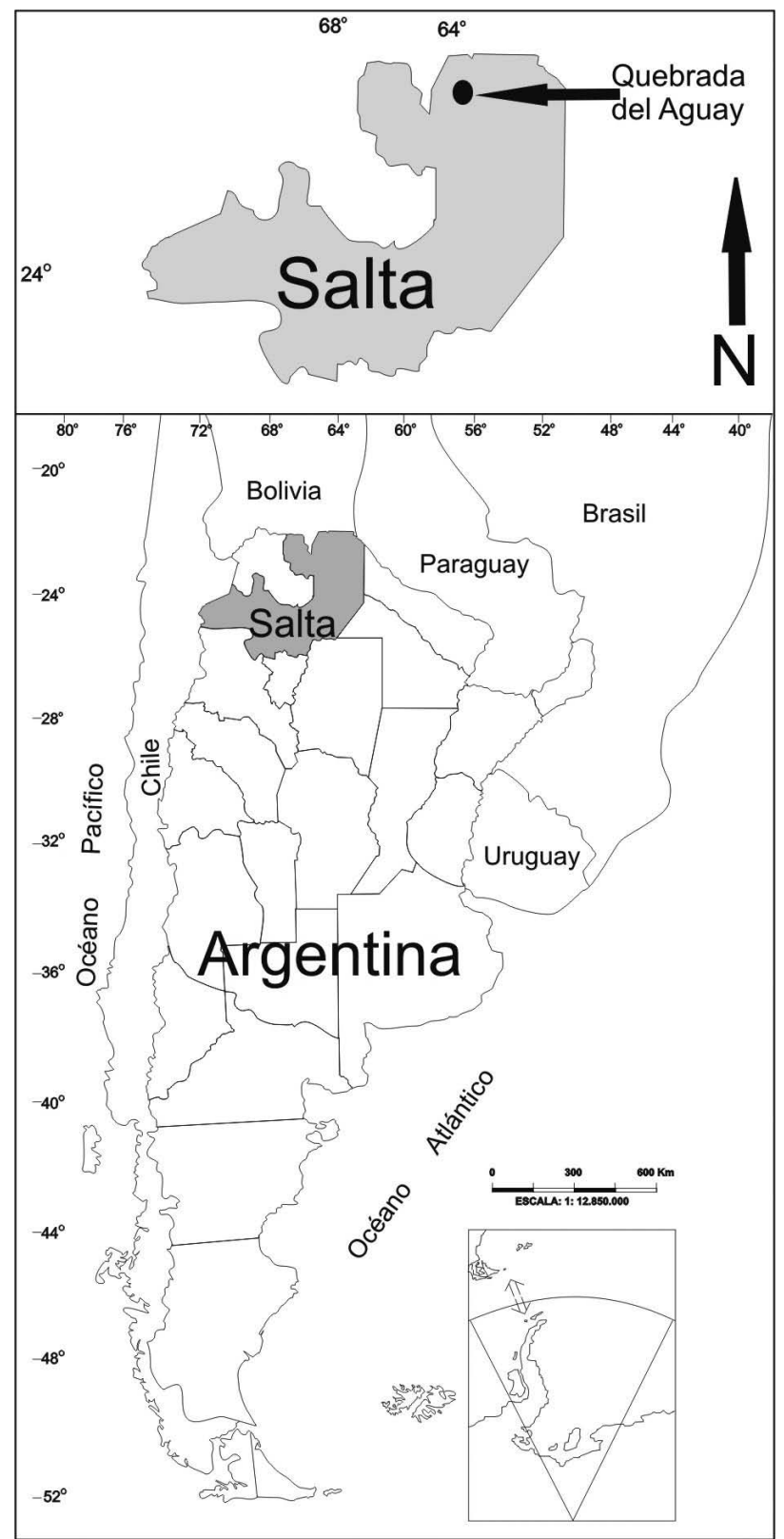

Figura 1.-Mapa de la Provincia de Salta (Argentina), el círculo indica la localidad fosilífera / Map of the Salta province (Argentina), the circle indicates the fossiliferous locality.

del Chaco y el Amazonas. Esta región se caracteriza por la presencia de montañas anticlinales y valles sinclinales, que producen remoción en masa como fenómeno general de evolución del relieve.

En general, los fósiles hallados en la Quebrada del Aguay provienen de sedimentitas rojizas, compuestas por areniscas, limo- arcilitas y arcilitas con óxido de hierro; algunas sedimentitas poseen carbonato de calcio. El ejemplar (PZ-MUPET 0116, Neosclerocalyptus sp.) fue hallado in situ, en limoarcilita rojiza clara, con signos incipientes de edafización y mayor con- 

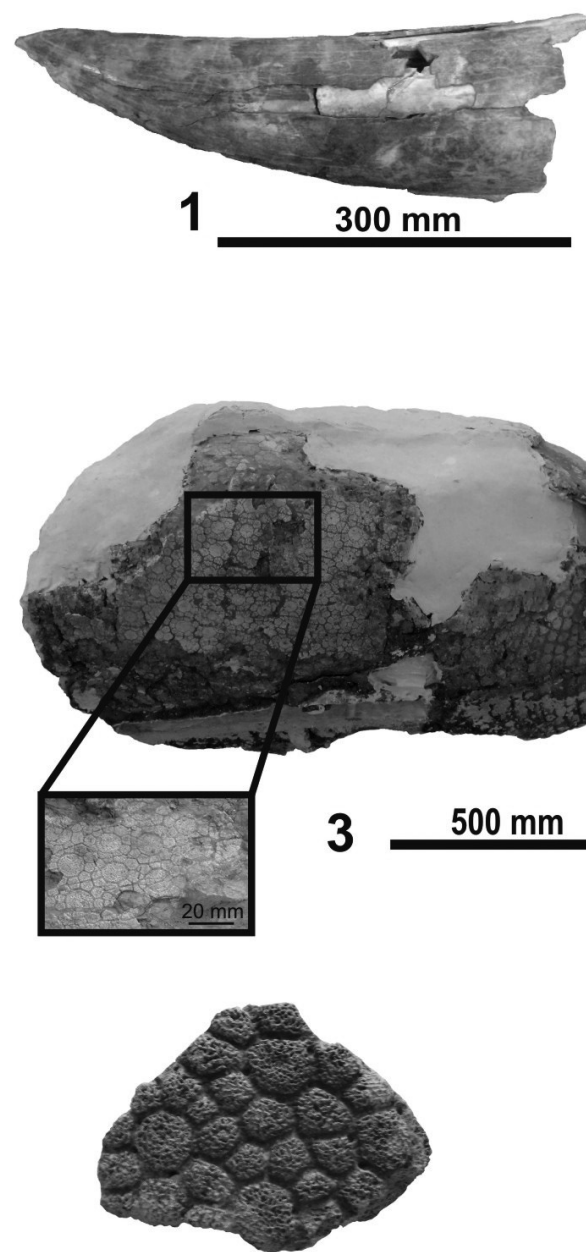

$510 \mathrm{~mm}$

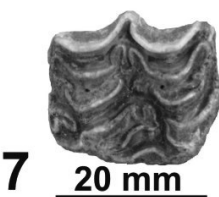

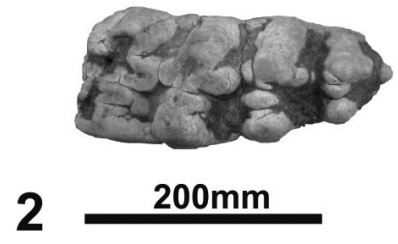

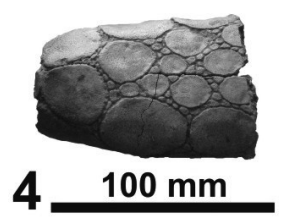

$500 \mathrm{~mm}$

Figura 2.-Stegomastodon sp. 1) (PZ-MUPET 0129) porción distal de un incisivo superior (defensa) /distal part of an upper incisive. 2) (PZ-MUPET 013) molariforme inferior en vista oclusal / lower molariform in oclusal view. Neosclerocalyptus sp. 3) (PZ-MUPET 0116) coraza dorsal en vista lateral derecha / dorsal carapace in lateral view. 4) (PZ-MUPET 0105) parte distal de tubo caudal en vista dorsal/distal part of caudal tube in dorsal view. Glyptodon sp. 5) (PZ-MUPET 0095) cuatro osteodermos asociados de la coraza dorsal en vista dorsal/four associated osteoderms of the dorsal carapace in dorsal view. Megatheriinae gen et sp. indet. 6) (PZ-MUPET 0201) molariforme en vista lateral / molariform in lateral view. Equus (Amerhippus) sp. 7) (PZ-MUPET 0202) M3? en vista oclusal/ M3? in oclusal view. Toxodon sp. 8) (PZ-MUPET 007) m3 en vista oclusal/ m3 in oclusal view.

tenido loessoide (Francisco Lucia com. per.), en tanto que otros ejemplares fueron hallados incluidos en conglomerado con matriz arenosa (PZ-MUPET 0095, Glyptodon sp.) o simplemente arenisca, mostrando cierto grado de transporte (PZMUPET 0129, PZ-MUPET 0131 Stegomastodon sp.; PZ-
MUPET 0201, Megatheriinae gen. et. sp. indet.; PZ-MUPET 0202, Equus (Amerhippus) sp.).

La secuencia litológica donde fueron hallados los fósiles mide, de base a techo, aproximadamente $15 \mathrm{~m}$. La base está compuesta por arenas friables que hacia arriba se vuelven más 


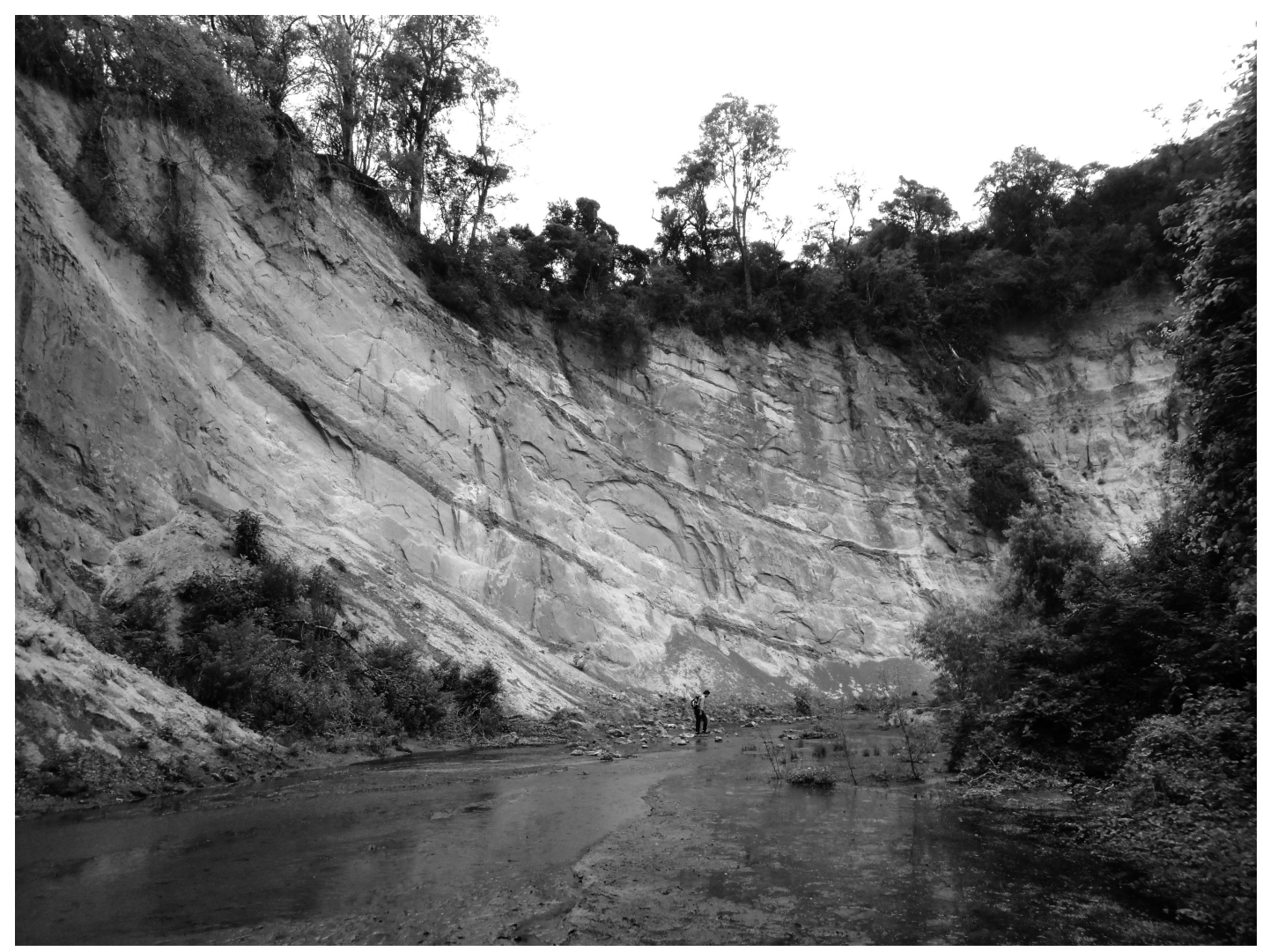

\begin{tabular}{|c|c|}
\hline$\left[\begin{array}{ccc}0 & 0 \\
0 & 0\end{array}\right.$ & Arena \\
\hline$\therefore \therefore$ & Arena más compacta \\
\hline $\mid, 1,1$ & 1. Limo- arcilita \\
\hline & Arcilita \\
\hline$O_{0}$ & Conglomerado \\
\hline 0 & Nódulos de carbonato de calcio \\
\hline
\end{tabular}

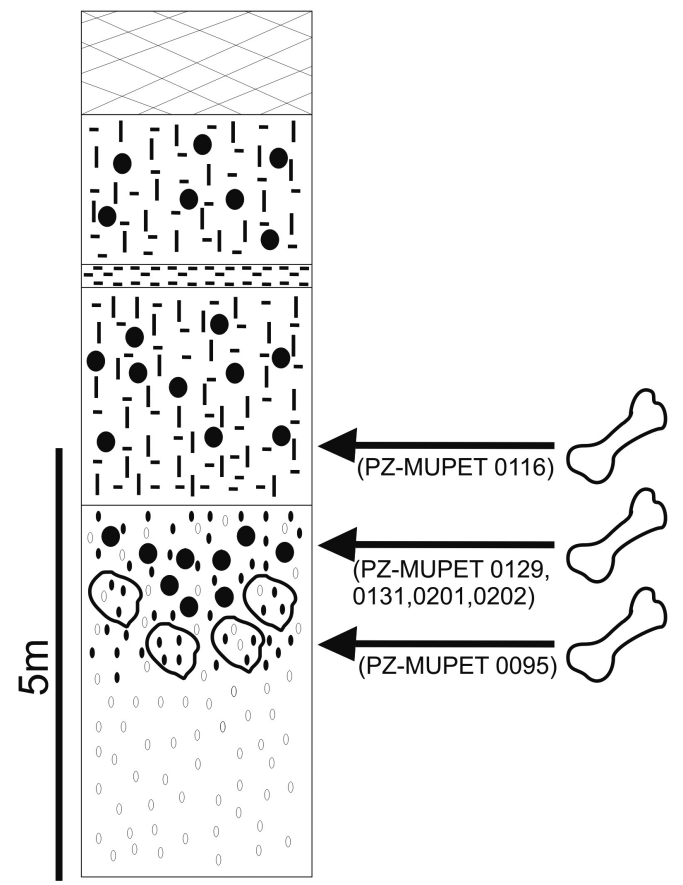

Figura 3.-1). Yacimiento Quebrada del Aguay, Salta, Argentina/ fossiliferous locality in Quebrada del Aguay, Salta province, Argentina 2) Perfil estratigráfico/ stratigraphical section. 
compactas, con intercalaciones de conglomerados de $100 \mathrm{~cm}$ de espesor aproximadamente; la secciones media y superior se caracterizan porque las sedimentitas cambian hacia limoarcilita intercalado lateralmente por una capa de $50 \mathrm{~cm}$ de arcilla pura muy consistente (Fig. 3).

De acuerdo con el trabajo de revisión sobre la geología del Cuaternario en Argentina realizado por Iriondo (2010), sumado a las caracteristicas litológicas de las sedimentitas presentes en la Quebrada del Aguay, la secuencia descripta podría corresponder a la Fm. Urundel (ver Iriondo, 1993). La edad estimada para esta unidad corresponde a $c a .36-8,5$ ka AP (Pleistoceno tardío-Holoceno temprano; Piso/Edad Lujanense).

\section{Sistemática paleontológica}

\author{
Orden Proboscidea Illiger, 1811 \\ Suborden Elephantoidea Osborn, 1912 \\ Familia Gomphotheriidae Cabrera, 1929 \\ Stegomastodon Pohlig, 1912 \\ Stegomastodon $\mathrm{sp}$.
}

Material referido y descripción: PZ-MUPET 0129, porción distal de un incisivo superior (defensa) en vista lateral; PZMUPET 0131, molariforme inferior cuadrangular y de gran tamaño, con cúspides cónicas a ambos lados y otras accesorias, que permiten que por desgaste se formen dos hileras (interna y externa) de figuras trifoliadas (ver Alberdi et al., 2002) (Fig. 2. 1 y 2).

Comentarios. De acuerdo con diversos autores el género Stegomastodon ingresó al continente sudamericano hace aproximadamente 2.6 Ma durante el apogeo del Gran Intercambio Biótico Americano (ver, entre otros, Alberdi et al., 2002; Prado et al., 2003; Alberdi \& Corona, 2005; Woodburne, 2010). Desde una perspectiva paleobiogeográfica, Prado et al. (2005) postulan la existencia de un corredor en América del Sur que permitió la dispersión del género, siguiendo las planicies costeras del actual territorio de Brasil. En Argentina, las provincias en donde se ha registrado la presencia del género son: Entre Ríos (Ferrero \& Noriega, 2009), Córdoba y Santa Fe (Alberdi \& Prado, 1995; Alberdi et al., 2008), Chaco (Zurita et al., 2004), Corrientes (Zurita \& Lutz, 2002), Santiago del Estero (Alberdi et al., 2008) y Buenos Aires (Cione et al., 2005); es precisamente en esta última donde alcanza su límite más austral.

Orden Cingulata Illiger, 1811

Suborden Glyptodontia Ameghino, 1889

Superfamilia Glyptodontoidea Gray, 1869

Familia Panochthidae Castellanos, 1927

Subfamilia Panochthinae Castellanos, 1927

Tribu Neosclerocalyptini Paula Couto, 1957

Neosclerocalyptus Paula Couto, 1957

Neosclerocalyptus sp.

Material referido y descripción: PZ-MUPET 0116, gran parte de una coraza dorsal, de forma ovalada, baja y alargada, con la parte anterior proyectada hacia delante en forma de "aletas". Los osteodermos son pequeños y delgados; la superficie dorsal presenta una figura central poco deprimida, rodeada por una hilera de figuritas poligonales, que se combinan entre osteodermos adyacentes, formando una trama donde son casi imperceptibles las suturas entre los osteodermos. Este conjunto de caracteres permite su asignación al género Neosclerocalyptus (ver Zurita, 2007); PZ-MUPET 0105, porción distal de un tubo caudal de contorno cilíndrico-cónico y de ornamentación similar a la de la coraza dorsal, ornamentado con un gran número de figuras ovales centrales rodeadas por una hilera de figuritas periféricas, características que permiten asignarlo al género Neosclerocalyptus (Zurita, 2007) (Fig. 2.3 y 4).

Comentarios. Fernícola (2008) sustenta la monofilia del suborden Glyptodontia en un marco cladístico y agrupa en la familia Panochthidae los géneros Neosclerocalyptus y Panochthus Burmeister, 1866. Neosclerocalyptus se ha registrado en Argentina, Paraguay, Uruguay y Bolivia (Zurita et al., 2005; 2009). En Argentina hay abundantes registros en la región chaco-pampeana y escasos en la región mesopotámica (Zurita et. al., 2002; Carlini et al., 2004). La distribución estratigráfica del taxón comprende el lapso Ensenadense- Lujanense (Pleistoceno temprano-Holoceno temprano) (Zurita, 2007).

Zurita et al. (2009) consideran a Neosclerocalyptus como un taxón adaptado a ambientes abiertos, áridos a semiáridos y fríos.

Familia Glyptodontidae Gray, 1869

Subfamilia Glyptodontinae Gray, 1869

Glyptodon Owen, 1838

Glyptodon sp.

Material referido y descripción: PZ-MUPET 0095, representado por numerosos osteodermos de la coraza dorsal. Estos presentan un notable espesor y la superficie expuesta muestra la típica ornamentación en "roseta" de los Glyptodontidae Glyptodontinae (Carlini et al., 2008). El surco anular que separa la figura central de las periféricas tiene un claro contorno en " $U$ ", con los lados paralelos entre si (Carlini et al., 2008). (Fig. 2. 5)

Comentarios. Carlini et al. (2008) establecen que la distribución latitudinal en América del Sur se extiende desde el sur de Argentina (provincia de Santa Cruz) hasta, probablemente, Colombia. Estudios realizados por Pérez et al. (2000) sobre el aparato hioideo de este género, sugieren que Glyptodon habría poseído hábitos pastadores.

Infraorden Pilosa Flower, 1883

Familia Megatheriidae Gray, 1821

Subfamilia Megatheriinae Gray, 1821

Megatheriinae gen. et. sp. indet.

Material referido y descripción: PZ-MUPET 0201, restos de molariformes. En vista oclusal, los molariformes presentan una sección cuadrada; estos caracteres permiten asignar el material a Megatheriinae gen. et. sp. indet. (ver De Iuliis et al., 2006; 2009) (Fig. 2. 6). 
Comentarios. Cartelle \& De Iuliis (2006) y De Iuliis et al. (2009) reconocen dos géneros de Megatheriidae, Megatherium Cuvier, 1796 y Eremotherium Spillman, 1948. El primero tiene una distribución restringida a América del Sur (De Iuliis et al., 2009) mientras que Eremotherium posee representantes que llegaron hasta América del Norte (Cartelle \& De Iuliis, 2006). Megatherium ha sido registrado en las regiones chaco-pampeana y mesopotámica (Zurita \& Lutz, 2002; Zurita et al., 2004, 2009; Carlini et al., 2004) en Argentina.

Orden Perissodactyla Owen, 1848

Familia Equidae Gray, 1821

Equus Linné, 1758

Equus (Amerhippus) Lund, 1840

Equus (Amerhippus) sp.

Material referido y descripción: PZ-MUPET 0202, constituido por M3? hipselodonte de forma subtriangular a ovalado, muy aplanado sobre su cara lingual, en donde se observa un surco mediano y separado del paracónulo por un istmo angosto. Esto permite su asignación a Equidae (Alberdi \& Prado, 2004). Más específicamente, la presencia en el molar superior de un protocono triangular y más alargado en su extremo distal que en la porción mesial, además de la presencia de plegamientos internos, permiten de acuerdo a Alberdi \& Prado (1992) su asignación a este subgénero (Fig. 2. 7).

Comentarios. El ingreso a América del Sur de la familia Equidae data de hace unos 2.6 Ma, coincidente con el apogeo del Gran Intercambio Biótico Americano (Webb, 1991; Woodburne, 2010). Se considera a los équidos sudamericanos como un grupo monofilético representado por dos géneros: Hippidion Spillman, 1931 y Equus (Amerhippus) Hoffstetter, 1950 (Alberdi \& Prado, 1993; Prado \& Alberdi, 1994). Según Alberdi \& Prado (1996) Hippidion es un género endémico de América del Sur cuyo registro fósil abarca desde el Plioceno superior al Pleistoceno tardío. Por el contrario, Equus (Amerhippus) es posterior, en tanto se registra en el Ensenadense de Tarija (Prado \& Alberdi, 1994) datado por MacFadden (2000) en 1.0-0.7 Ma. Su distribución geográfica va desde Colombia hasta la provincia de Buenos Aires (Argentina), y no se observa solapamiento de las distintas especies. En Argentina se han hallado restos asignados a Equidae en las regiones pampeana, mesopotámica y en la provincia de Chaco (Âlvarez, 1974; Carlini et al., 2004; Zurita et al., 2004). Los estudios sobre la morfología dentaria de los géneros de équidos sudamericanos realizados por Alberdi \& Prado (2004), postulan que Hippidion se alimentaba de material vegetal menos abrasivo que Equus (Amerhippus).

Orden Notoungulata Roth, 1903

Suborden Toxodonta Scott, 1904

Familia Toxodontidae Owen, 1845

Subfamilia Toxodontinae Trouessart, 1898

Toxodon Owen, 1837

Toxodon sp.

Material referido y descripción: PZ-MUPET 007, m3 con trigónido muy ancho y con la cara externa del talónido más plana, morfología que permite su asignación a Toxodon (MiñoBoilini et al., 2006). (Fig. 2. 8)

Comentarios. Las especies del género Toxodon se hallan entre los ungulados nativos de mayor talla y su registro es frecuente en sedimentos asignables al lapso Ensenadense-Bonaerense-Lujanense (Bond, 1999; Bond et al., 1995; Miño-Boilini et al., 2006). Bond et al. (1995) y Bond (1999) destacan que este género posee amplia distribución geográfica, abarcando los actuales territorios de Venezuela, Uruguay, Paraguay, Bolivia y Brasil, con registros en Argentina hasta el sur de la provincia de Buenos Aires. Czerwonogora et al. (2003) consideran a las especies de este género como formas pesadas y fundamentalmente pastadoras, infiriendo que vivían en ambientes abiertos.

\section{Discusión y conclusiones}

Como se ha mencionado en la introducción, los estudios referidos a la megafauna pleistocena en el noroeste de Argentina resultan muy escasos comparados con aquellos efectuados principalmente en la región Chaco-pampeana y mesopotámica (Cione \& Tonni, 1999; 2005; Zurita et al., 2009; Prado \& Alberdi, 2010). Para la provincia de Salta, esta es la primera asociación de mamíferos pleistocenos, ya que los aportes de Esteban et al. (1992), Carlini et al. (1999) y Zurita et al. (2002) mencionan taxones aislados.

La asociación faunística registrada Xenarthra Glyptodontidae, Glyptodon sp. y Panochthidae, Neosclerocalyptus sp; Tardigrada Megatheriidae gen. et. sp. indet.; Proboscidea Gomphotheriidae, Stegomastodon sp.; Notoungulata Toxodontidae, Toxodon sp. y Perissodactyla Equidae, Equus (Amerhippus) sp.) se encuentra conformada por taxones que han tenido durante el Pleistoceno amplia distribución latitudinal. Algunos de ellos, como Neosclerocalyptus, son indicadores de ambientes abiertos de pastizales y clima árido/semiárido, congruentes con un período glacial (Tonni et al., 1999).

Tonni et al. (1999; 2003) han sugerido, por ejemplo, que durante el Ultimo Máximo Glacial (OIS 2; ca. 18 ka AP), las temperaturas en la región Pampeana parecen haber sido de $2^{\circ} \mathrm{C}$ a $6^{\circ} \mathrm{C}$ más bajas que las imperantes en la actualidad, al tiempo que diversos autores han inferido, sobre la base de la distribución de arenas y loess, que las condiciones imperantes actualmente en la Patagonia podrían haberse extendido unos $750 \mathrm{~km}$ al noroeste de su límite actual (Iriondo y García, 1993).

En la actualidad, el área en donde fue hallado el elenco faunístico en estudio se encuentra compren- 
dida bajo el dominio Amazónico en la provincia de la Yunga (Cabrera, 1976), caracterizada por el desarrollo de selva pedemontana con regimenes de lluvia en el verano de 100-300 mm mensuales y en el invierno-primavera $10 \mathrm{~mm}$ (Brown et al., 2009). Durante el Pleistoceno tardío (OIS 2), el área en cuestión habría estado caracterizada por un ambiente en mosaico de bosques y áreas abiertas de pastizales (ver Cione et al., 2009). Esto resulta congruente con las condiciones climático-ambientales, fundamentalmente árido/semiárido, inferidas durante la sedimentación de la Fm. Urundel (ver Iriondo, 1993; 2010).

\section{AGRADECIMIENTOS}

Los autores desean expresar su agradecimiento al Prof. Alberto Julián Elicetche, a los Señores Tino Barrios, Amado Arena y la Sra. Andrea Tolaba, integrantes del Museo del Petróleo "Prof. Rodolfo Parodi Bustos" por habernos facilitado el material fósil para su estudio. Un particular agradecimiento a la familia Costas de la ciudad de Tartagal, provincia de Salta, (en especial a la señorita María Luisa Costas, por habernos comunicado sobre los hallazgos en Vespucio). Este agradecimiento se hace extensivo a los doctores Alicia Lutz, Ricardo Alonso, Martín Iriondo, Oscar Orfeo, Alberto Cione y a los licenciados Francisco Lucia y Edwin Chávez Aponte, por brindarnos desinteresadamente valiosa información para este estudio. Este agradecimiento se hace extensivo a los revisores, Dr. Daniel Perea y Dr. Edgardo Ortiz-Jaureguizar por las sugerencias efectuadas a la presente contribución. Este trabajo fue parcialmente financiado con los proyectos PICTO-UNNE $00164 \mathrm{y}$ F-011 (SGCyT-UNNE).

\section{Referencias}

Alberdi, M.T. \& Prado, J.L. (1992). El registro de Hippidion Owen, 1896 y Equus (Amerhippus) Hoffstetter, 1950 (Mammalia, Perissodactyla) en América del Sur. Ameghiniana, 29: 265-284.

Alberdi, M.R. \& Prado, J.L. (1995a). Los mastodontes de América del Sur. In: Evolución biológica y climática de la Región Pampeana durante los últimos 5 millones de años. Un ensayo de correlación con el Mediterráneo occidental (Alberdi, M.T.; Leone, G. \& Tonni, E.P., eds.) Monografías del Museo Nacional de Ciencias Naturales, CSIC, España, 12: 277-292.

Alberdi, M.T. \& Prado, J.L. (1995b). Los Équidos de América del Sur. In: Evolución climática y biológica de la región pampeana durante los últimos cinco millones de años. Un ensayo de correlación con el Mediterráneo occidental (Alberdi, M.T.; Leone, G. \& Tonni, E.P., eds.). Monografías del Museo Nacional de Ciencias Naturales, CSIC, España, 12: 295-308.

Alberdi, M.T. \& Prado, J.L. (2004). Caballos fósiles de América del Sur. Una historia de tres millones de años. INCUAPA (Investigaciones arqueológicas y paleontológicas del cuaternario), $269 \mathrm{pp}$.

Alberdi, M.T.; Cartelle, C. \& Prado, J.L. (2002). El registro de Stegomastodon (Mammalia, Gomphotheriidae) en el Pleistoceno superior de Brasil. Revista Española de Paleontología, 17: 217-235.

Alberdi, M.T.; Cartelle, C. \& Prado, J.L. (2003). El registro de Equus (Amerhippus) e Hippidion (Mammalia, Perissodactyla) de Brasil. Consideraciones paleoecológicas y biogeográficas. Ameghiniana, 40: 173-196.

Alberdi, M.T.; Cerdeño, E. \& Prado, J.L. (2008). Stegomastodon platensis (Proboscidea, Gomphotheriidae) en el Pleistoceno de Santiago del Estero, Argentina. Ameghiniana, 5: 257-271.

Álvarez, B.B. (1974). Los mamíferos fósiles del Cuaternario de Arroyo Toropí, Corrientes, Argentina. Ameghiniana, 11: 295-311.

Argollo, J. \& Iriondo, M.H. (Ed-) (2008). El Cuaternario de Bolivia y Regiones Vecinas. Museo Provincial de Ciencias Naturales Florentino Ameghino, Santa Fé, Argentina, 378 pp.

Bond, M.; Cerdeño, E.P. \& López, G. (1995). Los Ungulados Nativos de América del Sur. In: Evolución climática y biológica de la región pampeana durante los últimos cinco millones de años. Un ensayo de correlación con el Mediterráneo occidental (Alberdi, M.T.; Leone, G. \& Tonni, E P., eds.). Monografías del Museo Nacional de Ciencias Naturales, CSIC, España, 12: 259-275.

Bond, M. (1999). Quaternary native ungulates of Southern South America. A synthesis. Quaternary of South America and Antarctic Peninsula, 177-206.

Bandoni, D.; Soibelzon, E. \& Scarano, A. (2008). On Megatherium gallardoi (Mammalia, Xenarthra, Megatheriidae) and the Megatheriinae from the Ensenadan (lower to middle Pleistocene) of the Pampean region, Argentina. Geodiversitas, 30: 793-804.

Brown, A.D.; Bledinger, P.G.; Lomáscolo, T. \& García Bes, P. (Ed.). (2009). Selva Pedemontana de las Yungas. Historia natural, ecología y manejo de un ecosistema en peligro. Yerba Buena, Tucumán, Argentina, 449 pp

Burmeister, C.V. (1874). Monografía de los glyptodontes en el Museo Público de Buenos Aires. Anales del Museo Público de Buenos Aires, 35-42.

Cabrera, A.L. (1976). Regiones fitogeográficas de la República Argentina. Enciclopedia de Agricultura, Jardinería y Floricultura, Editorial Acme, Buenos Aires, 2: 1-85.

Carlini, A.A.; Tonni, E.P.; Alonso, R. \& Scillato Yané, G.J. (1999). Xenarthra (Mammalia) del Pleistoceno de Salta. $14^{\circ}$ Congreso Geológico Argentino, 1: 374-376.

Carlini, A.A.; Zurita, A.E.; Gasparini, G.M. \& Noriega, J.I. (2003). Los mamíferos del Pleistoceno de la Mesopotamia argentina y su relación tanto con aquellos del Centro-Norte de la Argentina, Paraguay, sur de Bolivia, como con los del sur de Brasil y oeste de Uruguay: Paleobiogeografía y Paleoambientes. INSUGEO Misceláneas, 12: 5-12.

Cartelle, C. \& De Iuliis, G. (2006). Eremotherium laurillar$d i$ (Lund) (Xenarthra, Megatheriidae), the anamerican 
giant ground sloth: taxonomic aspects of the ontogeny of skull and dentition. Journal of Systematic Palaeontology 4 (2): 199-209 6. doi:10.1017/S1477201905001781

Cione, A. \& Tonni, E.P. (1999). Biostratigraphy and chronological scale of upper-most Cenozoic in the Pampean Area, Argentina. Quaternary of South America and Antarctic Peninsula, 12: 23-51.

Cione, L.A. \& Tonni, E.P. (2005). Bioestratigrafía basada en mamíferos del Cenozoico superior de la provincia de Buenos Aires, Argentina. In: Relatorio del $16^{\circ}$ Congreso Geológico Argentino (De Barrio, R.E.; Etcheverry, R.O.; Caballé, M.F. \& Llambías, E., eds.) 11: 183-200.

Cione, A.L.; Tonni, E.P. \& Soibelzon, L. (2009). Did Humans Cause the Late Pleistocene-Early Holocene Mammalian Extinctions in South America in a Context of Shrinking Open Areas?. In: Haynes, G. (ed.). American Megafaunal Extinctions at the End of the Pleistocene. Springer Publishers, Vertebrate Paleobiology and Paleontology Series, Chapter,. EEUU: 125-144.

Cuvier, G. (1796). Notice sur le squelette d'une-très grande espèce de quadrupéde inconnue jusqu' à présent, trouvé au Paraguay, et déposé au Cabinet d'Histoire Naturelle de Madrid: Magasin Encyclopèdique, ou Journal des Sciences, des Lettres et des Arts, París, 2-3: 303-310.

Czerwonogora, A.; De Esteban-Trivigno, S. \& Fariña, R.A. (2003). Hábitos alimenticios de ungulados pleistocenos sudamericanos: un enfoque ecomorfológico. Ameghiniana (Suplemento), 40: 83.

De Iuliis, G. (2006). On the taxonomic status of Megatherium sundti Philippi, 1893 (Mammalia: Xenarthra: Megatheriidae). Ameghiniana, 43: 161-169.

De Iuliis, G.; Brandoni, D. \& Scillato-Yané, G.J. (2008). New remains of Megathericulus patagonicus Ameghino, 1904 (Xenarthra, Megatheriidae): information on primitive features of megatheriines. Journal of Vertebrate Paleontology, 28: 181-196.

De Iuliis, G.; Pujos, D. F. \& Guiseppe, T. (2009). Systematic and taxonomic revision of the Pleistocene ground sloth Megatherium (Pseudomegatherium) tarijense (Xenarthra: Megatheriidae). Journal of Vertebrate Paleontology, 29(4): 1244-1251.

Esteban, G.; Abdala, F. \& Nasif, N. (1992). Nuevos restos de Scelidotherium (Edentata) de Rosario de la Frontera, Provincia de Salta, Argentina. Consideraciones sistemáticas basadas en aspectos morfológicos del carpo. Boletín de la Real Sociedad Española Historia Natural, 87: 27-35.

Fernicola, J.C. (2008). Nuevos aportes para la sistemática de los Glyptodontia Ameghino 1889 (Mammalia, Xenarthra, Cingulata). Ameghiniana, 45: 553-574.

Ferrero, B.S. \& Noriega J. (2009). La paleontología de vertebrados en el Cuaternario de la provincia de Entre Ríos (Argentina): estado actual y perspectivas. Quaternário do Rio Grande Do Sul. Ribeiro, A.M.; Bauermann, S.G. \& Scherer, C.S. (eds.) Integrando conhecimentos. Monografías, Sociedad Brasileira de Paleontología, 1: 207-215.
Gervais, H. \& Ameghino, F. (1880). Los mamíferos fósiles de la América del Sud. Savy-Igon Hnos., París y Buenos Aires, 225 pp.

Gray, J.E. (1821). On the natural arrangement of vertebrose animals. London Medical Repository, 15: 296310.

Hoffstetter, R. (1950). Algunas observaciones sobre los caballos fósiles de América del Sur. Amerhippus gen. nov. Boletín Investigaciones Científicas Nacional, Quito Ecuador, 3: 426-454

Iriondo, M.H. (1993). La Formación Urundel, un loess chaqueño. In: Guía de Campo Provincia de Jujuy. $6^{\circ}$ Reunión de Campo de Cuaternario. CADINQUA/ Universidad Nac. Jujuy, 64: 67.

Iriondo, M.H. (2010). Geología del Cuaternario en Argentina. Editorial Moglia, Corrientes, Argentina, $437 \mathrm{pp}$.

Iriondo, M. \& García, N.O. (1993). Climatic variations in the Argentine plains during the last 18.000 years. Palaeogeography, Palaeoclimatology, Palaeoecology, 101: 209-220.

MacFadden, B.J. (2000). Middle Pleistocene Climate Change recorded in Fossil Mammal Teeth from Tarija, Bolivia, and Upper limit of the Ensenadan Land-Mammal Age. Quaternary Research, 54: 121-131. doi:10.1006/qres.2000.2146

Miño-Boilini, A.R.; Cerdeño, E. \& Bond, M. (2006). Revisión del género Toxodon Owen, 1837 (Notoungulata: Toxodontidae) en el Pleistoceno de las provincias de Corrientes, Chaco y Santa Fe, Argentina. Revista Española de Paleontología, 21: 93-103.

Owen, R. (1837). A description of the cranium of the Toxodon platensis, a gigantic extinct mammiferous species, referable by its dentition to the Rodentia, but with affinities to the Pachydermata and the herbivorous Cetacea. Proceedings Geological Society of London, 2: 541-542.

Owen, R. (1838). Fossil Mammalia (1), in Darwin, C. (ed.), The Zoology of the voyage of H.M.S. Beagle, under the command of Capitain Fitzroy, during the years 1832-1836. Londres, Smith Elder and Co., 1: 1$40 \mathrm{pp}$.

Paula Couto, C. (1957). Sôbre um gliptodonte do Brasil. Boletim Divisão de Geología e Mineralogía, 165: 1-37.

Pérez, L.M.; Scillato-Yané, G.J. \& Vizcaíno, S.F. (2000). Estudio morfofuncional del aparato hiodeo de Glyptodon cf. clavipes (Cingulata: Glyptodontidae). Ameghiniana, 37: 293-299.

Pérez, L.M.; Toledo, N.; De Iuliis, G. \& Bargo, M.S. (2010). Morphology and function of the hyoid apparatus of fossil Xenarthrans (Mammalia). Journal of Morphology, 271: 1119-1133.

Philippi, R.A. (1893). Vorla“ufige nachricht u“ ber fossile sa"ugethierknochen von Ulloma, Bolivia. Zeitschrift der Deutschen Geologischen, 45: 87-96.

Pohlig, H., (1912). Sur une vieille mandibule de "Tetracaulodon ohiotocum" Blum., avec défense in situ. Bulletin de la Société Belge Géologique, 26: 187-193.

Prado, J.L. \& Alberdi M.T. (2010). Changes of mammal assemblages in the pampean region (Argentina) and 
their relation with the Plio-Pleistocene boundary. Quaternary International, 212: 176-186. doi:10.1016/00310182(92)90140-Z

Saint-André, P.A. \& De Iuliis, G. (2001). The smallest and most ancient representative of the genus Megatherium Cuvier, 1796 (Xenarthra, Tardigrada, Megatheriidae), from the Pliocene of the Bolivian Altiplano. Geodiversitas, 23: 625-645.

Spillman, F. (1948). Beiträge zur Kenntnis eines neuen gravigraden Riesensteppentieres (Eremotherium caroliniense gen. et. sp. nov.), seines. Lebensraumes und seiner Lebensweise. Palaeobiologica, 8: 231-279.

Tonni, E.P.; Cione, A.L. \& Figini, A.J. (1999). Predominance of arid climates indicated by mammals in the pampas of Argentina during the Late Pleistocene and Holocene. Palaeogeography, Palaeoclimatology, Palaeoecology, 147: 257-281. doi:10.1016/S00310182(98)00140-0.

Tonni, E.P.; Huarte, R.; Carbonari, J.E. \& Figini, A.J. (2003). New radiocarbon chronology for the Guerrero Member of the Luján Formation (Buenos Aires, Argentina): palaeoclimatic significance. Quaternary International, 109-110: 45-48.

Tonni, E.P.; Soibelzon, E.; Cione, A.L.; Carlini, A.A.; Scillato Yané, G.J.; Zurita, A.E. \& Paredes Ríos, F. (2009). Preliminar correlation of the Pleistocene sequences of the Tarija valley (Bolivia) with the Pampean chronological standard. Quaternary International, 210: 57-65. doi:10.1016/j.quaint.2009.06.015.

Webb, S.D. (1991). Ecogeography and the Great American Interchange: Paleobiology, 17: 266-280.

Woodburne, M.O. (2010). The Great American Biotic Interchange: Dispersals, Tectonics, Climate, Sea Level and Holding Pens. Journal of Mammal Evolution, 17: 245-264. doi 10. 1007/s 10914-010-9144-8.
Zurita, A.E. (2007). Sistemática y evolución de los Hoplophorini (Xenarthra, Glyptodontidae, Hoplophorinae. Mioceno tardío-Holoceno temprano). Importancia bioestratigráfica, paleobiogeográfica y paleoambiental: Tesis doctoral, La Plata, Universidad Nacional de La Plata, 367 pp.

Zurita, A.E \& Lutz A.I. (2002). La fauna pleistocena de la Formación Toropí en la provincia de Corrientes (Argentina). Mastozoología Neotropical 9: 47-56.

Zurita, A.E.; Scillato-Yané, G.J.; Carlini, A.A. \& Alonso, R. (2002). Sclerocalyptus evidens Ameghino 1889 (Mammalia, Xenarthra, Glyptodontidae) en el Pleistoceno de Salta, Argentina. $8^{\circ}$ Congreso Argentino de Paleontología y Bioestratigrafía, resúmenes: 58.

Zurita, A.E.; Carlini, A.A.; Scillato-Yané, G.J. \& Tonni, E.P. (2004). Mamíferos extintos del Cuaternario de la provincia del Chaco (Argentina) y su relación con aquellos del este de la región pampeana y de Chile. Revista Geológica de Chile, 31: 65-89. doi: 10.4067/S0716-02082004000100004

Zurita, A.E.; Scillato-Yané G.J. \& Carlini, A.A. (2005). Palaeozoogeographic, biostratigraphic and systematic aspects of the genus Sclerocalyptus Ameghino 1891 (Xenarthra, Glyptodontidae) of Argentina. Journal of South American Earth Sciences, 20: 120-129. doi:10.1016/j.jsames.2005.06.013

Zurita, A.E., Miño-Boilini, A.R., Carlini A.A., Iriondo, M.H. \& Alcaraz, M.A. (2009) Paleontología del Chaco Oriental. Una nueva localidad con mamíferos fósiles pleistocenos del río Bermejo (Formosa, Argentina). Revista Mexicana de Ciencias Geológicas, 26: 277-288.

Recibido el 21 de octubre de 2010 Aceptado el 21 de marzo de 2011 Publicado online el 21 de noviembre de 2011 